Susanne Burren, Maritza Le Breton, Celestina Porta und Martin Böhnel

\title{
Zur Reproduktion von Differenz im Kontext von Internationalisierungsprozessen an Fach- hochschulen
}

Zusammenfassung: Der Beitrag beleuchtet institutionelle Bestrebungen zur Internationalisierung an Fachhochschulen und setzt diese in Bezug zu Erfahrungen migrantischer Studierender. Die Studie zu den Fachbereichen Soziale Arbeit, Pädagogik, Technik und IT sowie Wirtschaft zeigt, dass im Rahmen aktueller Programmatiken der Internationalisierung binäre Zuordnungen von «Eigen- und Fremdkultur» wirksam werden. Ähnliche Ein- und Ausgrenzungen manifestieren sich auch in Zuschreibungsprozessen, mit denen sich migrantische Studierende an den Hochschulen konfrontiert sehen. Unter dem Leitkonzept von Internationalisierung würden sich Möglichkeiten bieten, kulturalistische und andere essentialisierende Reproduktionsmuster von Differenz kritisch zu beleuchten. Jedoch lassen sich im Rahmen der Studie wenig Ansätze in diese Richtung erkennen.

Schlüsselwörter: Internationalisierung, Migrantische Studierende, Ungleichheits- und Differenzverhältnisse, Fachhochschulforschung

\section{About the reproduction of difference in the context of current implementations of internationalisation at universities of applied sciences}

Summary: The article examines institutional internationalisation efforts at universities of applied sciences and relates them to the experiences of migrant students. The study in the educational fields of social work, pedagogy, technology and IT as well as economics shows that binary classifications of "own" and "foreign" culture become effective in the context of current implementations of internationalisation. Similar processes of inclusion and exclusion manifest themselves in ascriptions with which migrant students are confronted at universities. The guiding concept of internationalisation would offer opportunities to critically examine culturalist and other essentialising patterns of reproducing difference. However, only a few approaches in this direction can be identified in the study.

Keywords: Internationalisation, Migrant students, Relations of inequality and difference, Research at universities of applied sciences 


\section{Einleitung}

Der Beitrag stellt Ergebnisse aus einem von 2016 bis 2019 durchgeführten Forschungsprojekt zur Internationalisierung an schweizerischen Fachhochschulen und damit verbundenen Ungleichheits- respektive Differenzverhältnissen vor. ${ }^{1}$ Charakteristisch für die aktuellen Internationalisierungsdynamiken an Fachhochschulen ist deren Beteiligung an länderübergreifenden Austauschprogrammen in Lehre und Forschung. Fachhochschulen bieten zudem in steigendem Masse Raum für Mitarbeitende und Studierende mit internationalem Herkunfts- und Bildungshintergrund. Allerdings findet dabei die vielfältige und facettenreiche Internationalität der Hochschulangehörigen wenig Beachtung. Gemäss Definition des Bundesamts für Statistik (2015) fällt in die Kategorie der «internationalen Studierenden", wer mit einem Hochschulzulassungsausweis aus dem Ausland an einer schweizerischen Hochschule eingeschrieben ist, wobei diese Gruppe als heterogen charakterisiert wird (ebd., S.9). Andere Begriffsbestimmungen wiederum fokussieren auf den Aspekt einer grenzüberschreitenden Mobilität zu Studienzwecken. Im vorliegenden Beitrag wird die Auffassung vertreten, dass sich die Internationalität von Studierenden nicht auf spezifische Zuwanderungsformen beschränken oder aufgrund des Migrationsstatus festlegen lässt. Internationalität in einem weiten Verständnis manifestiert sich an den Hochschulen gleichermassen durch Studierende, die sich selbst als migrantisch ${ }^{2}$ verstehen (bzw. von anderen so eingeordnet werden) wie durch die offiziellen Politiken und Programme der Hochschulen zur Internationalisierung. Davon ausgehend, setzt der vorliegende Beitrag die institutionellen Bestrebungen zur Internationalisierung an den Fachhochschulen in Bezug zu Erfahrungen, die von migrantischen Studierenden im Umgang mit den Voraussetzungen und Bedingungen des Hochschulstudiums gemacht werden. Im Beitrag werden diese zwei Dimensionen der Internationalisierung an Hochschulen beleuchtet und in beiden der Frage nachgegangen, inwiefern Differenzverhältnisse durch Mechanismen des «Zum-Anderen-machen» (Othering) reproduziert oder allenfalls kritisch aufgelöst werden. Das Konzept des “Othering” ist im Kontext der Postcolonial Studies zu verorten (Castro Varela 2016; Riegel 2016). Es bezeichnet binäre Unterscheidungspraktiken von «eigen» und «fremd», anhand derer asymmetrische Differenzordnungen hervorgebracht und dominante Wahrnehmungen geformt werden. Anders ausgedrückt wird somit also danach gefragt, inwiefern Otheringprozesse in den Internationalisierungsprogrammen der Hochschulen wirksam werden bzw. in den Berichten der Studierenden zum Ausdruck kommen und 
welche Analogien bzw. Unterschiede sich allenfalls zwischen beiden Bereichen zeigen.

Das Forschungsprojekt untersuchte hochschulische Rahmenbedingungen und Konsequenzen der Internationalisierung an Fachhochschulen in den vier ausgewählten Fachbereichen Soziale Arbeit, Pädagogik, Technik und IT sowie Wirtschaft und Dienstleistungen. Die Daten wurden an Fachhochschulen der deutsch- und französischsprachigen Schweiz erhoben. Datenbasis der Studie bilden zum einen Expert*inneninterviews mit Internationalisierungsverantwortlichen und Studiengangleitenden sowie Textdokumente zur Internationalisierung an den Hochschulen. Zum anderen wurden problemzentrierte Interviews mit migrantischen Studierenden in den Bachelor-Studiengängen der vier untersuchten Fachbereiche geführt.

Der Beitrag gliedert sich in zwei Hauptteile. Im ersten Teil werden theoretische Perspektiven zur Internationalisierung und deren Bedeutung für Differenz und Ungleichheit im Hochschulkontext diskutiert. Im zweiten Teil stehen die empirischen Ergebnisse der Studie im Zentrum: Zunächst werden Internationalisierungsverständnisse und -praktiken der Hochschulverantwortlichen auf deren Relevanz für Differenzverhältnisse hin beleuchtet. Anschliessend wird nachgezeichnet, wie Otheringprozesse für migrantische Studierende beim Zugang und im Verlauf ihres Studiums wirksam werden.

\section{Internationalisierung als Dimension der Hochschulbildung}

Die Internationalisierung der Hochschulen wird als ein Schlüsselmerkmal der Transformation tertiärer Bildung seit Ende der 1980er-Jahre beschrieben (Santiago et al. 2008). Knight definiert Internationalisierung als "the process of integrating an international, intercultural or global dimension into the purpose, functions or delivery of higher education" (2008, S. $7 \mathrm{f}$.). Sie geht von einem zweifachen Verständnis von Internationalisierung aus, dem jeweils unterschiedliche Aktivitäten zugeordnet sind: Internationalisierung "cross-border" beinhaltet die grenzüberschreitende Hochschulbildung resp. die Mobilität von Personen, Programmen, Wissen und Dienstleistungen über nationale Grenzen hinweg. Internationalisierung "at home” wiederum bezieht sich auf Aktivitäten am eigenen Campus einer Hochschule, wofür bspw. die Internationalisierung von Studienprogrammen und Lehrinhalten, die Entwicklung interkultureller Kompetenzen und Sprachkenntnisse von Mitgliedern einer Hochschule sowie die Integration von Internationalisierungsmassnahmen auf strategischer Ebene 
charakteristisch sind (ebd.). Im aktuellen Fachdiskurs zur Internationalisierung dominieren oftmals instrumentelle Sichtweisen, welche diese als proaktive Antwort der Hochschulen auf einen globalen Innovationswettbewerb einordnen. Die entsprechenden Überlegungen und Empfehlungen sind nicht nur generell durch ökonomische Argumente geprägt, sondern teilweise auch unmittelbar auf Profitinteressen ausgerichtet. Dies bspw., wenn sich die Aufmerksamkeit auf Möglichkeiten des Exports von Bildung in andere Länder sowie auf ausländische Studierende als neue Einnahmequelle für Hochschulen richtet (Jiang 2010; Teichler 2007).

Neben den breit vertretenen ökonomisch-instrumentell ausgerichteten Mainstreamansätzen im Internationalisierungsdiskurs finden sich auch kritischere Zugänge. So werden bspw. Konsequenzen der internationalen Bildungsmobilität für Klassenverhältnisse und Geschlechterungleichheiten diskutiert (Waters / Brooks 2011; Bauschke-Urban 2011) oder nachdrücklich auf die Vielfältigkeit studentischer Mobilität und damit verbundener Identitätsformen hingewiesen (Leung 2017; Raghuram 2012). In diesem Kontext wird vermehrt gefordert, Internationalisierung an Hochschulen neu zu denken. Womit sich auch die für die vorliegende Untersuchung relevante Frage verbindet, welche Studierenden unter welchen Umständen als «international» bzw. «einheimisch» zu bezeichnen sind (Madge et al. 2009, S. 34). Hu (2016) fordert dazu auf, die diesbezüglichen polaren Denkmodelle zu hinterfragen und Hochschulen als hybride kulturelle Begegnungs- und Diskursräume zu verstehen. Die nachgezeichneten Debatten diskutieren Internationalisierung in Hinblick auf soziale Hierarchisierungen und damit verbundene binäre Konzeptualisierungsformen. Migrantische Studierende und deren internationale Herkunftsund Bildungsbiografien bzw. transnationale Beziehungsnetzwerke (Pries 2008) sind allerdings sowohl in der Mainstreamliteratur zur Internationalisierung als auch im Rahmen dieser eher machtkritischen Zugänge bisher weitgehend unberücksichtigt geblieben.

\section{Differenz und Othering an Hochschulen}

Verschiedene Ansätze der Ungleichheitsforschung, die sich an den Theorien Bourdieus zur symbolischen Ordnung und Gewalt orientieren, begreifen Bildungsinstitutionen als Manifestations- und Reproduktionsorte sozialer Ungleichheit entlang sozialer, ethnischer oder geschlechtlicher Differenz (Arslan 2016). Symbolische Effekte sind gerade dann am wirksamsten, wenn die materiellen Machtstrukturen unsichtbar sind und die Dominanzverhältnisse naturalisiert werden. Dieser theoretische 
Hintergrund erlaubt u.a., subtile Differenz- und Ungleichheitserfahrungen als analytische Kategorien zu erfassen (Panagiotopoulou et al. 2016). Aktuell sind Hochschulen vermehrt als wichtige Instanzen sozialer Sortierung und symbolischer Grenzziehung in den Blick geraten, wobei auch die damit einhergehenden Bedingungen von Internationalisierung und Migration angesprochen werden (Pfaff-Czarnecka 2017; Mecheril / Klingler 2010; Darowksa / Machold 2010). Hierarchisierungen werden dabei insbesondere über Otheringprozesse wirksam. Gemäss Riegel (2016, S. 52f.) ist Othering in der postkolonialen Theoriebildung zentral für die Beschreibung von Prozessen des «Different-Machens», in denen im Zusammenspiel von hegemonialen Diskursen und Zuschreibungen resp. Essentialisierungen bestimmte Gruppen erst als solche hervorgebracht werden. Diese Konstruktionsprozesse erfolgen vor dem Hintergrund binärer Codierungssysteme, in denen das «Andere» als Negativfolie dient und symbolisch das von der Normalität Abweichende und Mangelhafte verkörpert. Somit verweist Othering auf die soziale Hierarchie zwischen dominanter «Wir»-Position und konstruierten "Anderen» (Saïd 1999). In Bezug auf Hochschul- bzw. Bildungsinstitutionen bedeutet dies auch, dass die formale bzw. institutionelle Ebene eine machtvolle Rolle spielt: Hochschulen legen formale Anforderungen fest, um die Zulassung zu regeln (bspw. Praxiserfahrung oder Sprachkompetenzausweise für Studierende, deren Erstsprache nicht der Hochschulsprache entspricht) oder sie identifizieren Studierende, welche für Unterstützungsmassnahmen berechtigt sind (bspw. Nachteilsausgleich für Studierende mit einer Beeinträchtigung). Der Anspruch bleibt grundsätzlich verwehrt, wenn die festgelegten Bedingungen nicht erfüllt bzw. nachgewiesen werden.

\section{Die Relevanz der Sprache}

Im Hinblick auf Internationalisierung im Hochschulkontext und Differenzerfahrungen migrantischer Studierender kommt der Sprache als wichtiges Element symbolischer Ordnung eine besondere Bedeutung zu (Kalpaka 2005; Darowksa / Machold 2010). So wird der monolinguale Habitus als diskriminierender Aspekt von Bildungsinstitutionen bezeichnet (Heinemann / Dirim 2016; Gogolin 2008) sowie im Kontext globaler Bildungsprozesse auf Dynamiken der Rekolonisierung verwiesen (Castro Varela / Heinemann 2016). Wie Arslan (2016) anmerkt, wird Mehrsprachigkeit an deutschen Hochschulen mit prestigeträchtigen Sprachen wie Englisch in Zusammenhang gebracht, während Minderheitssprachen wie bspw. Türkisch kaum Beachtung finden. Fabricius et al. (2017) heben hervor, dass 
Internationalisierungsbestrebungen der Hochschulen zu paradoxen Effekten in Hinblick auf Sprachkompetenzen führen. Diese gehen nicht mit der Förderung sprachlicher Pluralität einher, vielmehr bestätigt sich eine Tendenz zur Vereinheitlichung entlang hegemonialer Ordnungsmuster. Internationalisierung ist an den Hochschulen somit oftmals in erster Linie "Englishization" (Hu 2016, S. 264). Damit werde ein enormes Potenzial für eine differenzierte Wissensentwicklung und entsprechende Lehr- und Lernpraktiken nicht ausgeschöpft.

\section{Internationalisierung an Schweizer Fachhochschulen}

Das hier vorgestellte Forschungsprojekt eruierte einerseits das Verständnis von Internationalisierung an Fachhochschulen sowie die damit verbundenen Umsetzungsformen. Andererseits wurde gefragt, welche institutionellen Hürden und Optionen sich für migrantische Studierende beim Zugang und im Verlauf ihres Studiums ergeben. Wie sich zeigt, werden im Rahmen aktueller Programmatiken der Internationalisierung binäre Zuordnungen von «Eigen-» und «Fremdkultur» wirksam, die sich in ähnlicher Weise auch in den Erfahrungen migrantischer Studierender widerspiegeln. Zudem verweisen die Untersuchungsresultate unter Bezug auf Gutiérrez Rodríguez et al. (2016, S. 163) darauf, dass gängige Verständnisse von Internationalisierung «nicht auf nachhaltige Gleichstellung in der Migrationsgesellschaft» abzielen.

\section{Methodisches Vorgehen}

Das methodische Vorgehen der Studie gliedert sich in zwei Bereiche. Das an Fachhochschulen der Deutsch- und Westschweiz erhobene Datenmaterial basiert zum einen auf insgesamt 17 Expert*inneninterviews (Gläser / Laudel 2009) mit Zuständigen für Internationalisierung sowie mit Studiengangleitenden aus den vier Fachbereichen Soziale Arbeit, Pädagogik, Technik und IT sowie Wirtschaft und Dienstleistungen. Zudem wurden sowohl auf Hochschul- wie auch auf nationaler Ebene die für Internationalisierung relevanten Textdokumente erfasst. Es handelt sich hierbei um rund 70 Policy-Dokumente (Strategien, Leitlinien) sowie Textbeiträge, die zur Kommunikation mit den Studierenden dienen. Die Auswertung dieser Daten erfolgte mit einem Verfahren, das sich an der qualitativen Inhaltsanalyse nach Kuckartz (2012) orientiert und zudem Elemente des Theoretischen Kodierens der Grounded Theory übernimmt (Strauss / Corbin 1996).

Zum anderen umfasst die Studie 31 Interviews mit migrantischen Studierenden im Bachelor-Studium aus den untersuchten Fachbe- 
reichen. Ausgewählt wurden die befragten Studierenden nach inhaltlichen bzw. soweit möglich entlang von kontrastierenden Kriterien, wie bspw. Geschlecht, Migrations- und Bildungshintergrund, Alter, Sprache, Hochschulstandort, Studienmodus und Semesteranzahl. Diese Daten wurden mittels Theoretischem Kodieren in Anlehnung an die Grounded Theory (Strauss / Corbin 1996; Glaser/ Strauss 2005) ausgewertet, wobei die Verdichtung anhand mehrerer Teilschritte erfolgte. Zuerst wurden die Daten offen kodiert und Fallcharakterisierungen erstellt, anschliessend erfolgte eine Komprimierung der Daten entlang der für die Fragestellung relevanten Dimensionen. In einem letzten Schritt wurden diese thematischen Dimensionen anhand einer vertikalen Analyse zueinander in Verbindung gesetzt.

\section{Internationalisierung als Förderung von Studierendenmobilität}

Wie die Interviews mit Hochschulverantwortlichen und die Dokumentenanalyse verdeutlichen, sind institutionelle Bestrebungen, die gegenwärtig an den Fachhochschulen unter dem Leitbegriff der Internationalisierung stattfinden, unterschiedlich weit fortgeschritten bzw. in vielen Fällen noch wenig konsolidiert. Internationalisierung wird in verschiedenen Hochschuldokumenten wie bspw. im Fachbereich Soziale Arbeit zwar als «integraler Bestandteil des Leistungsauftrags» (Dokument 65, S. 8) bezeichnet, die Umsetzung dieser Programmatiken steht jedoch nicht selten noch in den Anfängen.

In dieser Aufbauphase zeigen sich aber bereits deutliche Übereinstimmungen in den Zielsetzungen. So sind die Aktivitäten im Bereich Internationalisierung an allen untersuchten Hochschulen in erster Linie darauf ausgerichtet, grenzüberschreitende studentische Mobilität zu organisieren und zu fördern. Dies bspw. indem den Studierenden ein Semesteraufenthalt an einer Partneruniversität im Ausland ermöglicht wird. Wie die Aussage des Experten L. Marzino ${ }^{3}$ verdeutlicht, gilt studentische Auslandsmobilität als unhinterfragter Standard von Internationalisierung: «Studierende müssen mobil sein, [...] das ist per se international» (Z. 173). Entsprechend wird an der Hochschule für Wirtschaft, an welcher Marzino tätig ist, in den nächsten fünf Jahren mit einer Verdoppelung der Mobilitätsnachfrage gerechnet und die damit verbundene Zielsetzung ist es, Mobilität durchgehend in die Studienpläne zu integrieren. Gemäss dem weiter oben erläuterten Doppelverständnis von Internationalisierung fokussieren die bisherigen Internationalisierungsprogramme somit weitgehend auf "cross-border"-Aktivitäten. Hingegen finden Massnahmen zur Internationalisierung "at home" deutlich weniger Beachtung resp. wird diese 
als zu entwickelndes Handlungsfeld beschrieben und erscheint damit in Bezug auf Inhalte und Zielsetzungen noch unbestimmt.

Migrantische Studierende bleiben im Kontext der Internationalisierungsbestrebungen unerwähnt. Auch auf Nachfrage hin bekunden die Expert*innen Mühe, einen Zusammenhang zwischen den Themenbereichen Internationalisierung und Migration herzustellen. Ansätze, Internationalisierung umfassender zu verstehen, zeigen sich bspw. bei der Studiengangleiterin im Fachbereich Wirtschaft, N. Umaga, die darauf verweist, dass für eine «wirklich internationale» Hochschule viel mehr Studierende mit «verschiedenen Backgrounds» notwendig seien. «So neunzig Prozent von meinen Studenten sind alle Schweizer und das heisst, wir haben wirklich nicht so viele Unterschiede - so Migrationshintergrund oder internationaler Hintergrund.» (ebd., Z. 7-9)

Die einseitige Fokussierung auf Mobilitätsförderung im Rahmen der Internationalisierungsprogramme könnte den Blick auf eine differenziertere Sichtweise internationaler Verhältnisse an Fachhochschulen verstellen. Diese sind heute nämlich wesentlich durch die Entwicklung hin zur Migrationsgesellschaft geprägt: Migrantische Studierende sind an schweizerischen Fachhochschulen zwar unterrepräsentiert, sie machen aber doch - über alle Fachbereiche hinweggesehen - 29 Prozent aller Studierenden aus, wobei die Tendenz steigend ist (BFS 2017). Dies bedeutet, dass sich - von der Auslandsmobilität während des Studiums einmal abgesehen - Internationalität in unterschiedlichen Formen bei den Studierenden manifestiert. In den institutionellen Internationalisierungsprogrammen finden aber entsprechende Ressourcen wie bspw. Mehrsprachigkeit und / oder internationale Erfahrungen migrantischer Studierender kaum Berücksichtigung.

\section{Rekurs auf Kulturalisierung im Internationalisierungsdiskurs}

Ein verbreitetes Internationalisierungsmotiv setzt programmatisch voraus, dass Auslanderfahrungen die Kompetenzen im Umgang mit Interkulturalität erweitert. Dabei wird oftmals ausgeblendet, dass solche Kompetenzerweiterungen nicht selbstläufig erfolgen, sondern einer reflektierten, theoriebasierten Auseinandersetzung bedürfen (Massumi 2017). Für den Studiengangleiter im Fachbereich Wirtschaft, L. Strewe, sollen die Bestrebungen zur Internationalisierung zwar in erster Linie die Arbeitsmarktfähigkeit der Studierenden verbessern. Als «Seiteneffekt» möchte er diese aber auch dazu befähigen, «im Umgang mit unterschiedlichsten Kulturen Verantwortung übernehmen zu können für das Handeln, das man 
im interkulturellen Kontext hat.» (Z. 18-21) Wie Darowska und Machold (2010) kritisch festhalten, impliziert das Alltagsverständnis von Interkulturalität einen statischen Kulturbegriff, der auf die Markierung von Fremdheit und Nicht-Zugehörigkeit hindeutet. In diesen Vorstellungen wird Kultur in homogenisierender Weise als Einheit und zugleich als wesenhafte Eigenschaft verstanden, die Anderssein begründet, während mögliche multiple Zugehörigkeiten ausser Acht bleiben. Zudem werden kulturelle Unterschiede oftmals ohne plausible Begründung mit Nation, Ethnie oder einem geografischen Territorium gleichgesetzt (ebd., S. 20, vgl. auch Kalpaka 2005).

Die Aussagen der befragten Fachpersonen verweisen auf essentialisierende und homogenisierende Konzepte kultureller Zugehörigkeit. Dies sowohl wenn von "incoming students" die Rede ist, welche die Hochschule im Rahmen der Auslandsmobilität besuchen, als auch hinsichtlich als migrantisch attribuierter Studierenden. Entsprechende Differenzmarkierungen werden im Datenmaterial an pauschalisierenden Gruppenzuordnungen wie "die Schweizer», "die Chinesin», "die Ausländer» deutlich. Ein Studiengangleiter spricht von «Ić-Studenten», um Studierende zu bezeichnen, denen er aufgrund ihres Familiennamens eine Herkunft aus «Ex-Jugoslawien» zuschreibt (T. Althaus, Z. 650 f.). An anderer Stelle werden Studierende als Repräsentant*innen «ihrer» Nation adressiert oder es wird ihnen anhand von binären Unterscheidungen kulturelle Andersheit zugeschrieben. Dies bspw., wenn es um die Präsenz von Austauschstudierenden in Lehrveranstaltungen und studentischen Projektgruppen geht. In diesem Kontext führt die Internationalisierungsverantwortliche M. Dedo an, dass Studierende, die keine Studienaufenthalte im Ausland absolvieren, «kulturell von diesen Leuten [den Austauschstudierenden] profitieren würden» (Z. 56). Ähnlich argumentiert B. Rosinus, ein Beauftragter für Internationalisierung im Fachbereich Technik:

In diesen Projekten versuchen wir, diesen Projektgruppen eben ab und zu auch einen "Incoming» reinzutun, damit die mal ein wenig eine Horizonterweiterung erhalten [...]. Also ich meine, das ist dann der Benefit von unseren eigenen Studierenden, die nicht selber in einen Austausch gehen. (Z. 56-60)

Beide Expert*innen konnotieren kulturelle Unterschiede positiv und deuten diese grundsätzlich als Bereicherung. Gemäss der binären Logik von «eigen» und «fremd» werden solche Unterschiede jedoch als gegeben vorausgesetzt und damit essentialisiert. «Einheimische» und “Incomings” ste- 
hen sich als zwei scheinbar homogene Gruppen gegenüber, wobei Letztere in der Tendenz als nicht-zugehörig markiert werden.

Wie die Daten verdeutlichen, nehmen Hochschulverantwortliche öfters Bezug auf Alltagsverständnisse über den «Austausch der Kulturen» und kaum auf aktuelle wissenschaftliche Diskurse, in denen sich kulturalisierungskritische Ansätze bereits seit längerem durchgesetzt haben. Im Kontext der derzeitigen Debatte um eine differenzsensible Hochschullehre wird dazu aufgefordert, Differenzordnungen als konstitutiver Bestandteil von Bildungsprozessen bewusst «in Un-Ordnung» zu bringen (Hoffarth et al. 2013, S. 52), um soziale Kategorisierungen und damit einhergehende Identifizierungspraktiken deutlich zu machen. Dies wirft die Frage auf, wie Internationalisierungsmassnahmen im Bereich der Hochschullehre mit einer kritischen Reflexion von Differenzzuschreibungen verknüpft werden könnten.

\section{Zuschreibung von Andersheit migrantischer Studierender in der monolingualen Hochschule}

In den Begründungen für die Internationalisierung bzw. die Förderung von Auslandsmobilität lassen sich implizite Konzepte kultureller Differenz erkennen, an welche sich dann wiederum diskursive Mechanismen des «Zum-Anderen-machen» im Sinne von Othering anschliessen können. Wie im Folgenden ausgeführt wird, sind ähnliche Reproduktionsmuster auch für die Erfahrungen migrantischer Studierender an Fachhochschulen kennzeichnend. Die Interviews mit migrantischen Student*innen verweisen auf stereotype Zuschreibungen und stigmatisierende Hürden aufgrund von Differenz. Sie erlauben zudem Einblick in Ausdrucksweisen von Andersheit, mit denen sich diese Studierenden an der Hochschule konfrontiert sehen bzw. worauf sie sich selbst beziehen.

Am Beispiel der Sprachanforderungen für die Zulassung zum Studium werden Otheringprozesse als unhinterfragte Normalität an den untersuchten Hochschulen in beiden Sprachregionen sichtbar. So thematisiert die Studentin S. Erdal an einer Deutschschweizer Hochschule in Bezug auf das Zulassungsverfahren, dass im Fachbereich Soziale Arbeit - neben einem sechsmonatigen Praktikum - der Nachweis von Deutschkenntnissen auf Niveau C2 des europäischen Referenzrahmens für Sprachen ${ }^{4}$ als Aufnahmebedingung vorausgesetzt wird. Dies wird ihrerseits als exkludierend wahrgenommen, da der Besuch von Sprachkursen im Vorfeld des Studiums hohe Kosten verursacht. Darüber hinaus absolvierte S. Erdal zur 
Erweiterung ihrer Sprachkenntnisse zusätzliche Praktika, woraus für sie neben dem finanziellen ein zeitlicher Mehraufwand entstand:

Dafür muss man viel Geld investieren, damit man diesen Abschluss bekommen kann. Dafür muss man viele Sprachkurse machen, also das finde ich eine hohe Hürde. (Z. 53-55)

An einer Pädagogischen Hochschule in der Westschweiz thematisiert eine migrantische Studentin mit Erstsprache Deutsch den für angehende Primarlehrpersonen im ersten Studienjahr obligatorischen Französisch-Test. Gemäss Studienreglement attestiert die Prüfung den Studierenden die «Beherrschung von Französisch als Unterrichtssprache» und eine Fortsetzung des Studiums ist nur mit bestandener Prüfung möglich. Die Studentin C. Durion berichtet, dass dadurch Professionalität sichergestellt würde und meint damit, «jeden Sachverhalt und Kommunikation klar rüberzubringen in Französisch»(Z. 259-260). Die Anforderung C2 für Studienanwärter*innen nicht deutscher Erstsprache, welche die Studienberechtigung nicht in deutscher Sprache erworben haben, setzt für das Studium so genannt «muttersprachliche» Kenntnisse voraus, die wiederum mit perfekten Sprachkenntnissen gleichgesetzt werden. Dabei wird nicht mitbedacht, dass die Wissenschaftssprache Deutsch bzw. Französisch von allen Studierenden unabhängig von ihrer Erstsprache zu erlernen ist (Knappik / Dirim 2013).

Monolinguale Selbstverständnisse werden umgekehrt auch von Studierenden reproduziert. So betrachtet sich C. Durion als angehende Primarlehrerin in Bezug auf den Unterricht in ihrer Erstsprache Deutsch gegenüber ihren Mitstudierenden aus der Westschweiz als kompetenter:

Sprache ist ja nicht nur reine Grammatik, sondern da kommt ja mehr mit, da kommt Kultur, sei es deutsche [...]. Aber einfach was so mit deutscher Kulturgeschichte noch zusammenhängt [...], aber dass ich da einfach authentisch bin und ich dann eine gute Lehrerin sein kann. (C. Durion, Z. 542-549)

Die Sequenz verdeutlicht, wie sie sich selbst essentialisiert, denn ihres Erachtens kann sie aufgrund ihrer Erstsprache deutsche Kulturgeschichte «authentischer» bzw. wirklichkeitsnäher vermitteln als ihre französischsprachigen Mitstudierenden. Sie verschliesst damit aber den Blick auf absolvierte Studienleistungen und Praxiserfahrungen, also auf im Rah- 
men ihrer Bildungs- und Berufsbiografie erworbene Ressourcen und Kompetenzen.

Kritische Forschungsperspektiven monieren defizitäre Sichtweisen auf migrationsspezifische Mehrsprachigkeit aufgrund monolingualer Selbstverständnisse (Knappik / Dirim 2013; Kalpaka 2009). Dies widerspiegelt sich in den Zulassungsanforderungen der untersuchten Fachhochschulen. Sprachliche Zulassungsvoraussetzungen auf Niveau C2 zeigen sich in den Fachbereichen Soziale Arbeit und Pädagogik als weitgehend unhinterfragte Norm. So wird die Konstruktion des "Anderen» aufgrund sprachlicher Anforderungen verfestigt. Dies bestätigt sich über sprachregionale Grenzen hinweg, indem die jeweils dominante regionale Erstsprache zur Norm wird, um nach formalen Kriterien zwischen Studierenden zu unterscheiden.

In den Fachbereichen Wirtschaft und Technik erfahren die Sprachanforderungen an den untersuchten Hochschulen eine etwas andere Gewichtung. Im Fachbereich Wirtschaft muss abhängig von den Bedingungen der jeweiligen Hochschulen für die Studienzulassung maximal das Sprachniveau C1 gemäss europäischem Referenzrahmen ausgewiesen werden. Im Fachbereich Technik gilt das Sprachniveau B2 als ausreichend. Umgekehrt ist das Studienangebot in englischer Sprache in diesen beiden Fachbereichen deutlich stärker ausgebaut und der Status von Englisch als «Lingua franca» weitgehend unhinterfragt. So wird bspw. an einer Hochschule für Wirtschaft festgehalten, dass «die Anforderungen einer zunehmenden Internationalisierung zu erfüllen, u.a. gute Englischkenntnisse bedingt» (Dokument 51, S. 4). Diese hohe Bewertung der englischen Sprache stellt zwar eine Alternative zur monolingualen Hochschule dar, setzt aber gleichzeitig das Muster der fehlenden Anerkennung für migrationsbedingte Mehrsprachigkeit fort. Während Englisch als «Schlüssel zur Welt» eine hohe Gewichtung erfährt, werden alle anderen Herkunftssprachen implizit abgewertet (Krüger-Potratz 2016, S. 34). Insoweit wird Mehrsprachigkeit weiterhin als migrationsspezifisches Wissen dequalifiziert.

\section{Othering aufgrund des Aussehens und kulturellen Zuschreibungen}

Otheringprozesse kommen an den untersuchten Hochschulen ebenso in Bezug auf people of colour, Aussehen und religiöse Symbole zum Ausdruck. So dient das äussere Erscheinungsbild für Dozierende als Motiv, den Studierenden aufgrund binärer Konstruktionslogiken Andersheit zu unterstellen. Bei der Studentin Z. Anesani wird deren Hautfarbe sowie der von ihr getragene Turban von einigen Dozierenden als Anlass genommen, sie 
als Repräsentantin einer anderen Kultur zu markieren und ihr mangelnde Deutschkenntnisse zuzuschreiben. Als Studentin im Fachbereich Soziale Arbeit legt Anesani dar, wie ihr auf Hochdeutsch die Frage gestellt wurde, ob sie die Ausführungen in der Lehrveranstaltung nachvollziehen könne:

Verstehen Sie mich? Und nachher, das ist so spannend, dass ein Akademiker nicht davon ausgeht, dass jemand, die so aussieht wie ich, auch hier sozialisiert werden kann. Als hätte er bereits eine feste Vorstellung im Kopf von Menschen mit anderer Hautfarbe. (Z. 227-230)

Auf eine ähnlich defizitäre Betrachtungsweise verweist A. Panou, die berichtet, wie ihr von einer Dozentin vorgehalten wurde, der Lehrveranstaltung nicht folgen zu können, weil sie «Ausländerin» sei. Die Studentin schildert, wie ihr aufgrund vermeintlicher Verständigungsschwierigkeiten in stereotypisierender Weise kulturelle Andersheit unterstellt wurde:

Ich ging nach dem Unterricht zu ihr, und sie [die Dozentin] hat mir ein Etikett gegeben. Ja du, du bist Ausländerin, deshalb weisst du nicht (...). (Z. 409-410)

Die Kontrastierung der Fachbereiche verweist auf Konfigurationen von Andersheit aufgrund von Sprache, race und äusserem Erscheinungsbild. Die Schilderungen der Studierenden thematisieren Situationen, in denen diskursiv zugeschriebene körperliche oder kulturelle Merkmale als herabwürdigende Unterscheidung zwischen «uns» und den «anderen» Wirkung entfalten. Dabei lassen sich Kontinuitäten beobachten, welche «kulturelle Andersheit» als Abweichung von der Mehrheitsgesellschaft betrachten. Dies zeigt sich über alle Fachbereiche hinweg in ähnlicher Weise. Dies obwohl bspw. gerade in der Sozialen Arbeit der Auftrag darin bestünde, «essentialisierende Perspektiven und stigmatisierende Effekte» kritisch zu hinterfragen (Mecheril / Melter 2010, S. 126).

\section{Fazit}

Die Aktivitäten und Programme zur Entwicklung von Internationalität sind prinzipiell auf Mobilitätsförderung ausgerichtet und reproduzieren die binäre Logik von «internationalen» und «einheimischen» Studierenden. Damit gerät die Komplexität internationaler Verhältnisse an Hochschulen tendenziell aus dem Blick. Gleichzeitig eröffnet sich über implizite Konzepte der kulturellen Differenz ein Raum für Fremdheitszuschreibungen, die von den befragten Expert*innen in unterschiedlicher Weise wahr- 
genommen werden. Die Erfahrungen migrantischer Studierender verweisen auf Ein- und Ausgrenzungsprozesse aufgrund unterschiedlicher Achsen der Differenz. Hierbei zeigen sich institutionelle Hürden insbesondere auch im Bereich der tendenziell monolingualen Verhältnisse im Fachhochschulkontext und den damit verbundenen formalen Anforderungen. Aus dieser Perspektive verdeutlichen die Daten, wie binäre Differenzordnungen sowohl seitens der institutionellen Akteur*innen als auch der Studierenden in ähnlicher Weise reproduziert werden.

Die Ausklammerung migrationsgesellschaftlicher Zugänge aus dem Themenfeld der Internationalisierung veranschaulicht Widersprüche und Paradoxien pädagogischen Handelns im tertiären Bildungskontext sowie die fehlende Beachtung von Ressourcen und Potenzialen migrantischer Studierender (Karakaşoğlu 2016). Insbesondere im Bereich der Internationalisierung «at home» würden sich für Hochschulen Möglichkeiten bieten, differenzreflexive Perspektiven in die Curricula einzubringen. Entsprechende Ansätze implizieren sowohl die Frage nach einer kritischen Auseinandersetzung mit Differenzverhältnissen im Kontext von Internationalisierung als auch die Bereitschaft, Ausgrenzungsprozessen auf der symbolischen und strukturellen Ebene entgegenzutreten. Die Ergebnisse der hier vorgestellten Studie lassen jedoch wenig Entwicklungen in diese Richtung erkennen.

Die Untersuchung weist auf Möglichkeiten hin, Internationalisierung an Hochschulen in ihren unterschiedlichen Facetten zu verstehen und zugrundeliegende Machtstrukturen ins Blickfeld zu rücken. Die Studie führt Forschungsansätze zur Internationalisierung von Hochschulen mit theoretischen Perspektiven auf gesellschaftliche Differenzordnungen zusammen und verschränkt damit Dimensionen der Internationalisierungsdynamik, die in der Literatur bisher noch kaum auf diese Weise ausgelotet wurden. Dieser Zugang ermöglicht, Leerstellen wie jene der Vernachlässigung migrationsgesellschaftlicher Verhältnisse an Hochschulen sowie die Kontinuität von Otheringprozessen gegenüber migrantischen Studierenden sichtbar zu machen.

\section{Literatur}

Arslan, Emre (2016). Reflexion eines Projektes zur Mehrsprachigkeit in der universitären symbolischen Ordnung. In:
Emre Arslan \& Kemal Bozay (Hrsg.), Symbolische Ordnung und Bildungsungleichheit in der Migrationsgesellschaft. Wiesbaden: Springer VS, S. 501-522. 
Bauschke-Urban, Carola (2010). Hochschulen zwischen Transnationalisierung und Provinzialität. Intersektionelle Perspektiven. In: Carola Bauschke-Urban, Marion Kamphans \& Felizitas Sagebiel (Hrsg.), Subversion und Intervention. Wissenschaft und Geschlechter(un)ordnung. Opladen/Farmington Hills:

Barbara Budrich, S. 243-261.

Bundesamt für Statistik BFS (2015). Internationale Studierende an den Schweizer Hochschulen. Themenbericht der Erhebung 2013 zur sozialen und wirtschaftlichen Lage der Studierenden. Neuchâtel.

Bundesamt für Statistik BFS (2017). Studienund Lebensbedingungen an den Schweizer Hochschulen. Neuchâtel.

Castro Varela, María Do Mar (2016). Postkolonialität. In: Paul Mecheril (Hrsg.), Handbuch Migrationspädagogik. Weinheim und Basel: Beltz, S. 152-166.

Castro Varela, María do Mar \& Heinemann, Alisha M. B. (2016). Globale Bildungsbewegungen - Wissensproduktionen verändern. In: Zeitschrift für internationale Bildungsforschung und Entwicklungspädagogik. Münster/New York/München/Berlin: Waxmann, S. 17-22.

Darowska, Lucyna \& Machold, Claudia (2010). Hochschule als transkultureller Raum unter den Bedingungen von Internationalisierung und Migration eine Annäherung. In: Lucyna Darowska, Thomas Lüttenberg \& Claudia Machold (Hrsg.), Hochschule als transkultureller Raum? Kultur, Bildung und Differenz in der Universität. Bielefeld: transcript, S. 13-38.

Fabricius Anne H.; Mortensen, Janus \& Haberland, Hartmut (2017). The lure of internationalization: paradoxical discourses of transnational student mobility, linguistic diversity and crosscultural exchange. In: Higher Education, 73, S. 577-595.

Glaser, Barney G. \& Strauss, Anselm L. (2005). Grounded Theory. Strategien qualitativer Forschung. Bern: Huber.
Gläser, Jochen \& Laudel, Grit (2009). Experteninterviews und qualitative Inhaltsanalyse. Wiesbaden: Springer VS.

Gogolin, Ingrid (2008). Der monolinguale Habitus der multilingualen Schule. Münster/New York/München/Berlin: Waxmann.

Gutiérrez Rodríguez, Encarnación; Ha, Kien Nghi; Hutta, Jan; Kessé, Emily Ngubia; Laufenberg, Mike \& Schmitt, Lars (2016). Rassismus, Klassenverhältnisse und Geschlecht an deutschen Hochschulen. Ein runder Tisch, der aneckt. In: $s u b$ urban. Zeitschrift für kritische Stadtforschung, 4(2-3), S. 161-190.

Heinemann, Alisha M. B. \& Dirim, Ínci (2016). «Die sprechen bestimmt (schlecht) über mich». Sprache als ordnendes Prinzip im Bildungssystem. In: Emre Arslan \& Kemal Bozay (Hrsg.), Symbolische Ordnung und Bildungsungleichheit in der Migrationsgesellschaft. Wiesbaden: Springer VS, S. 199-214.

Hoffarth, Britta; Klingler, Birte \& Plösser, Melanie (2013). Reizende Ereignisse. Irritation als Beunruhigung und als Verschiebung von Ordnungen. In: Paul Mecheril, Birte Klingler, Britta Hoffarth, Claudia Machold, Margarete Menz, Melanie Plösser, Nadine Rose, Susann Fegter \& Susanne Arens (Hrsg.), Differenz unter Bedingungen von Differenz. Zu Spannungsverhältnissen universitärer Lehre. Wiesbaden: Springer VS. S. 51-70.

$\mathrm{Hu}$, Adelheid (2016). Internationalisierung und Mehrsprachigkeit: Universitäten als interkulturelle und mehrsprachige Diskursräume. In: Almut Küppers, Barbara Pusch \& Pinar Uyan Semerci (Hrsg.), Bildung in transnationalen Räumen. Wiesbaden: VS, S. 257-268.

Jiang, Xiaoping (2010). Towards the internationalisation of higher education from a critical perspective. In: Journal of Further and Higher Education, 32(4), S. 347-358.

Kalpaka, Annita (2005). Pädagogische Professionalität in der Kulturalisierungsfalle - Über den Umgang mit «Kultur» in Verhältnissen von Differenz und Dominanz. In: Rudolf Leiprecht \& Anne 
Kerber (Hrsg.), Schule in der Einwanderungsgesellschaft. Ein Handbuch. Schwalbach am Taunus: Wochenschau Verlag, S. 387-405.

Kalpaka, Annita (2009). Institutionelle Diskriminierung im Blick - Von der Notwendigkeit Ausblendungen und Verstrickungen in rassismuskritischer Bildungsarbeit zu thematisieren. In: Wiebke Scharathow \& Rudolf Leiprecht (Hrsg.), Rassismuskritik. Band 2: Rassismuskritische Bildungsarbeit. Schwalbach am Taunus: Wochenschau Verlag, S. 25-40.

Karakaşoğlu, Yasemin (2016). Hochschule. In: Paul Mecheril (Hrsg.), Handbuch Migrationspädagogik. Weinheim/Basel: Beltz, S. 386-402.

Knappik, Magdalena \& Dirim, Ínci (2013). "Native-Speakerism" in der Lehrerbildung. In: Journal für LehrerInnenbildung, 3, S. 20-23.

Knight, Jane (2008). Internationalisation: Key Concepts and Elements. In: Michael Geabel, Lewis Purser \& Bernd Wachter (Hrsg.), Internationalisation of European Higher Education. Berlin/Stuttgart: Josef Raabe, S. 1-22.

Krüger-Potratz, Marianne (2016). Migration als Herausforderung für öffentliche Bildung. In: Aysun Doğmuş, Yasemin Karakaşoğlu \& Paul Mecheril (Hrsg.), Pädagogisches Können in der Migrationsgesellschaft. Wiesbaden: Springer VS, S. 13-41.

Kuckartz, Udo (2012). Qualitative Inhaltsanalyse. Methoden, Praxis, Computerunterstützung. Weinheim/Basel: Beltz.

Leung, Maggi W. H. (2017). Social mobility via academic mobility: reconfigurations in class and gender identities among Asian scholars in the global north. In: Journal of Ethnic and Migration Studies, 43(16), S. 2704-2719.

Madge, Clare; Raghuram, Parvati \& Noxolo, Patricia (2009). Engaged pedagogy and responsibility: A postcolonial analysis of international students. In: Geoforum, 40, S. 34-45.
Massumi, Mona (2017). Internationale Mobilität ohne reflexive Mobilität? Eine rassismuskritische Auseinandersetzung mit studienbezogenen Auslandsaufenthalten in der Lehrer_innenbildung. In: Karim Fereidooni \& Meral El (Hrsg.), Rassismuskritik und Widerstandformen. Wiesbaden: Springer VS, S. 573-587.

Mecheril, Paul \& Melter, Claus (2010). Differenz und Soziale Arbeit. Historische Schlaglichter und systematische Zusammenhänge. In: Fabian Kessl, Melanie Plösser (Hrsg.), Differenzierung, Normalisierung, Andersheit. Soziale Arbeit als Arbeit mit den Anderen. Wiesbaden: Springer VS, S. 117-131.

Mecheril, Paul \& Klingler, Birte (2010). Universität als transgressive Lebensform. Anmerkungen, die gesellschaftliche Differenz- und Ungleichheitsverhältnisse berücksichtigen. In: Lucyna Darowska, Thomas Lüttenberg \& Claudia Machold (Hrsg.), Hochschule als transkultureller Raum? Kultur, Bildung und Differenz in der Universität. Bielefeld: transcript, S. 83-116.

Panagiotopoulou, Argyro; Rosen, Lisa \& Wagner, Matthias (2016). Die Frage ist halt jetzt, darf Pippi Langstrumpfs Vater noch der Negerkönig sein. Zur (Re-)Produktion von Rassismus im Rahmen universitärer Lehrveranstaltungen. In: Emre Arslan \& Kemal Bozay (Hrsg.), Symbolische Ordnung und Bildungsungleichheit in der Migrationsgesellschaft. Wiesbaden: Springer VS, S. 239-256.

Pfaff-Czarnecka, Johanna (2017). Einleitung. Universitäten - als Orte der Heterogenität und Un/Gleichheit. In: Joanna PfaffCzarnecka (Hrsg.), Das soziale Leben der Universität. Studentischer Alltag zwischen Selbstfindung und Selbstbestimmung. Bielefeld: transcript, S. 11-42.

Pries, Ludger (2008). Die Transnationalisierung der sozialen Welt. Sozialräume jenseits von Nationalstaaten. Frankfurt am Main: Suhrkamp.

Raghuram, Parvati (2012). Theorising the Spaces of Student Migration. In: Population, Space and Place, 19, S. 138-154. 
Riegel, Christine (2016). Bildung - Intersektionalität-Othering. Pädagogisches Handeln in widersprüchlichen Verhältnissen. Bielefeld: transcript.

Saïd, Edward (1999). Die Konstruktion des «Anderen». In: Burgmer Christoph (Hrsg.), Rassismus in der Diskussion. Berlin: Elefanten Press, S. 27-44.

Santiago, Paulo; Tremblay, Karine; Ester, Basri \& Arnal, Elena (2008). Internationalisation: Shaping Strategies in the National Context. In: Tertiary Education for the Knowledge Society 2, S. 235-309.
Strauss, Anselm \& Corbin, Juliet (1996). Grounded Theory: Grundlagen Qualitativer Sozialforschung. Weinheim: Beltz.

Teichler, Ulrich (2007). Die Internationalisierung der Hochschulen. Neue Herausforderungen und Strategien. Frankfurt am Main / New York: Campus.

Waters, Johanna \& Brooks, Rachel (2011). International/transnational spaces of education. In: Globalisation, Societies and Education, 9(2), S. 155-160.

\section{Anmerkungen}

1 Das vom Schweizerischen Nationalfonds finanzierte Projekt «Internationalisierung an Fachhochschulen: Zur Bedeutung von Geschlecht und Migration für Bildungs(un)gleichheit» wurde in Kooperation mit der Hochschule für Soziale Arbeit (HSA), der Pädagogischen Hochschule (PH) und der Hochschule für Angewandte Psychologie (APS) der Fachhochschule Nordwestschweiz (FHNW) durchgeführt. Neben den in diesem Beitrag vorgestellten qualitativen Untersuchungsbereichen beinhaltet es auch eine Studierendenbefragung (Vollerhebung) in den vier untersuchten Fachbereichen.

2 Das Adjektiv «migrantisch» steht hier als Synonym für die Bezeichnung «Migra- tionshintergrund» und bezieht sich auf eine Selbstpositionierung im Kontext von Emanzipationsbestrebungen. Dieses Attribut ist - im Gegensatz zur angeblichen homogenen Herkunft der Mehrheitsgesellschaft - offen für sämtliche Phänomene, die in relativ unbestimmter Form mit Migration in Bezug stehen (Polat et al. 2014).

3 Das gesamte Datenmaterial wurde anonymisiert. Französisch- und englischsprachige Aussagen wurden für den vorliegenden Beitrag übersetzt.

4 Der Referenzrahmen umfasst die Niveaus A1 für «Anfänger» bis C2 für «annähernd muttersprachliche Kenntnisse». Vgl. https://www.europaeischerreferenzrahmen.de/.

\section{Biografische Angaben}

Susanne Burren, Dr. rer. soc., ist Senior Researcher und Leiterin Gleichstellung und Diversity an der Pädagogischen Hochschule der FHNW. Nach einem Lizentiat in Ethnologie hat sie in Soziologie promoviert. Ihre Arbeitsschwerpunkte liegen in den Bereichen Hochschul- und Wissenschaftssoziologie, Gender Studies und Diversitäts- forschung. Ihre neueren Arbeiten beschäftigen sich mit unterschiedlichen Aspekten von Ungleichheit im (höheren) Bildungswesen.

Maritza Le Breton, Dr. phil., ist Professorin am Institut Integration und Partizipation der Hochschule für Soziale Arbeit FHNW. Sie ist Sozialwissenschaftlerin und beschäftigt sich seit vielen Jahren mit Geschlechter-, Diversity- und Mig- 
rationsforschung. Ihre Arbeiten widmen sich geschlechter-, migrations- und bildungssoziologischen Fragestellungen, insbesondere den Ursachen und Mechanismen von Geschlechterungleichheit im globalen Kontext am Beispiel von Frauenhandel und Sexarbeit sowie Differenzverhältnissen aufgrund von Migration und Geschlecht - und anderen Strukturkategorien - im Fachhochschulkontext.

Celestina Porta, M.A., ist seit 2012 als wissenschaftliche Assistentin bzw. Mitarbeiterin an der Pädagogischen Hochschule FHNW tätig. Bis 2016 engagierte sie sich an der Professur Interkulturalität und Sozialisationsprozesse des Instituts Primarstufe, seither am Institut Forschung und Entwicklung. Ihre Arbeitsschwer- punkte liegen in der Migrations-, Gender- und Diversitätsforschung.

Martin Böhnel, Mag. M. Sc., hat 2010 einen Magisterabschluss in Internationaler Entwicklung an den Universitäten Wien und Lyon erworben und 2016 einen Master of Science in Sozialer Arbeit an der Zürcher Hochschule für Angewandte Wissenschaften ZHAW. Er verfügt über Berufserfahrung in der internationalen Entwicklungszusammenarbeit und Non-Profit Organisationen insbesondere zu Themen der Migration. Seit Herbst 2016 ist er als wissenschaftlicher Assistent am Institut Integration und Partizipation der Hochschule für Soziale Arbeit FHNW mit den Themenschwerpunkt Bildung im Kontext von Migration und Gender tätig. 